\title{
Beware We Are Erupting As Siamese Twins
}

Srinivasan $\mathrm{K}^{1} \&$ Chitra $\mathrm{S}^{2}$

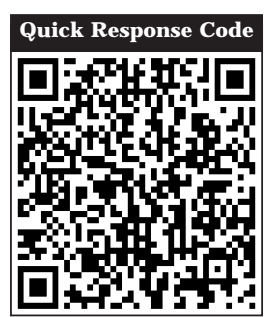

doi: $10.5866 / 2015.7 .10222$

'Senior Lecturer,

Department of Pedodontics And Preventive Dentistry, Adhiparasakthi Dental College And Hospital,

Melmuruvathur, Tamil Nadu.

${ }^{2}$ Associate Professor,

Department of Anaesthesia,

Chirstian Medical College, Vellore,

Tamil Nadu.

\section{Article Info:}

Received: J uly 11, 2015

Review Completed: August 11, 2015

Accepted: September 12, 2015

Available Online: J uly, 2015 (www.nacd.in)

(c) NAD, 2015 - All rights reserved

\section{Email for correspondence:}

skskskpedo@gmail.com

\begin{abstract}
:
Anomalies of the dentition present real challenges to the Dental Practitioner. Various terms have been used to describe Dental twinning anomalies: Gemination, F usion, Concrescence, Double teeth, Conjoined teeth, Twinned teeth, Geminifusion and Vicinifusion. Dental anomalies of number and forms may occur both in the primary and permanent Dentition. Fusion is a condition in which the crowns of two separate teeth have been joined together during the crown development. Fusion occurs infrequently but could cause esthetic, spacing and periodontal problems. It may requirea hard multidisci plinary approach with Pedodontist, Orthodontics, Endodontics, Surgery and Prosthetics to solve aesthetic and functional problems.

The present case report highlights the presence of a primary double tooth in an 8-year-old girl involving both primary right maxillary central and lateral incisor with a discussion of clinical implications and various treatment methods. Cases with primary double tooth necessitate careful examination as they may be associated with anomalies in the succeeding permanent dentition and require proper treatment planning.
\end{abstract}

Key words: Aesthetics, Developmental anomaly, Diagnosis, Fused Primary I ncisors, Gemination, Radiographic evaluation.

\section{Introduction:}

The anomaly of conjoined teeth has been described under a variety of names. 'Connate' was one of the first introduced terminologies by Tomes (1859). Later, De J onge (1955) proposed the terms 'Schizodontia' to describe teeth, which originate by partial division of Dental anlage and 'Synodontia' for those formed by the inability of adjacent tooth germs to retain their individuality. Although the term double teeth, suggested by Miles (1954) seems to be more widely accepted and is more appropriate if the etiology is not known. ${ }^{1-5}$

Tooth fusion is defined as union between the dentin and or enamel of two or more separate developing teeth.1, 2 The fusion may be partial or total depending upon thestage of tooth development at the time of union, a distinguishing feature between fusio-totalis, partialis-coronaries and 
partialis-radicularis. ${ }^{3,4}$ If the contact occurs before the calcification stage, the teeth unite completely and form one large tooth. Incomplete fusion may be at root level if the contact and union occurs after formation of crown. ${ }^{2}$ The present case report highlights the presence of a primary double tooth in an 8 year old girl involving both primary right maxillary central and lateral incisor.

\section{Case Report:}

An 8-year-old girl along with her mother, presented to my Dental office at Vellore.

The parental concern was doubleteeth in upper front teeth that caused aesthetic and psychological problems. The patient's mother was concerned about the effect the "double tooth" which might have upon the child's permanent teeth. The child was asymptomatic upon presentation and the mother reported no past Dental consultation was sought. Clinical examination revealed a child with height and weight normal for her age and no physical abnormalities. There was no family history of Dental anomalies and no consanguinity was reported in the parents.

Intraoral examination revealed the following (Figure 1)

- The maxillary dental arch presented one large primary central incisor that was abnormally wide and had bifid crown.

- Theincisors showed a buccal and palatal groove from the incisal edge to the cervical portion of the tooth and were not affected either by dental caries or periodontal problems.

- The maxillary right side deciduous central and lateral incisors were fused together.

- The patient exhibited normal age specific dentition with class I occlusion. The rest of the dentition was normal without any variations or anomalies.

No discomfort was present on vertical and horizontal percussion or on palpation in the surrounding area. Thermal and el ectric pulp testing was done on left lateral incisor, right maxillary lateral incisor. The patient and the parent were informed about the delayed eruption of permanent central and incisor.
An oral investigation revealed the erupting tooth with regard to normal dentition. The periapical radiograph exhibited that the crowns and the roots were fused with complete union of their pulp chambers and presence of single pulp chamber and root canal, which is one of the distinguishing features of geminated teeth from fusion. The roots of fused primary teeth had not resorbed. As a general rule, when the affected tooth is regarded as one, if in the arch there is one tooth less than the normal count it is called fusion, while when the normal number of teeth is present it is termed gemination [Duncan and Helpin,1987].

The treatment plan was explained to her family and with their consent. No dental restorations were evident and the periodontal status was satisfactory. Prior to this, no dental consultation was sought. Treatment was recommended in order to prevent periodontal disease, development of dental caries, to improve the esthetic status of the patient and to provide a normal eruptive pathway for canine.

Initially the fused teeth were separated in the coronal level using long, thin diamond bur. After this, an elevator was used to try and separate the supernumerary tooth from the lateral incisor. However, this was not successful. Extraction of fused teeth was planned. Treatment procedure was initiated by administering local anesthesia (Lignocaine Hydrochloride Anhydrous 21.3mg, 2\% w/v, Epinephrine 1:200,000, Neon, India) in the upper labial sulcus and palatal area in the region of 51 to 52. Extraction of fused teeth was carried out and periodic review was advised. The extraction socket was inspected for any pathology. Patient was kept on antibiotic, anti-inflammatory and analgesic regimen for 5 days. The oral hygieneinstruction was then given to the patient (Figure 2 and 3). The patient recovered uneventfully in a couple of days. Eruption of the maxillary right central and lateral incisor and other permanent teeth was found to be normal. The final appearance of the teeth was esthetically pleasing with gingival margins at the same level.

\section{Discussion:}

Fusion is a rare Dental developmental anomaly. ${ }^{5}$ Double tooth is a term used to describe connate tooth and includes both Dental fusion and 

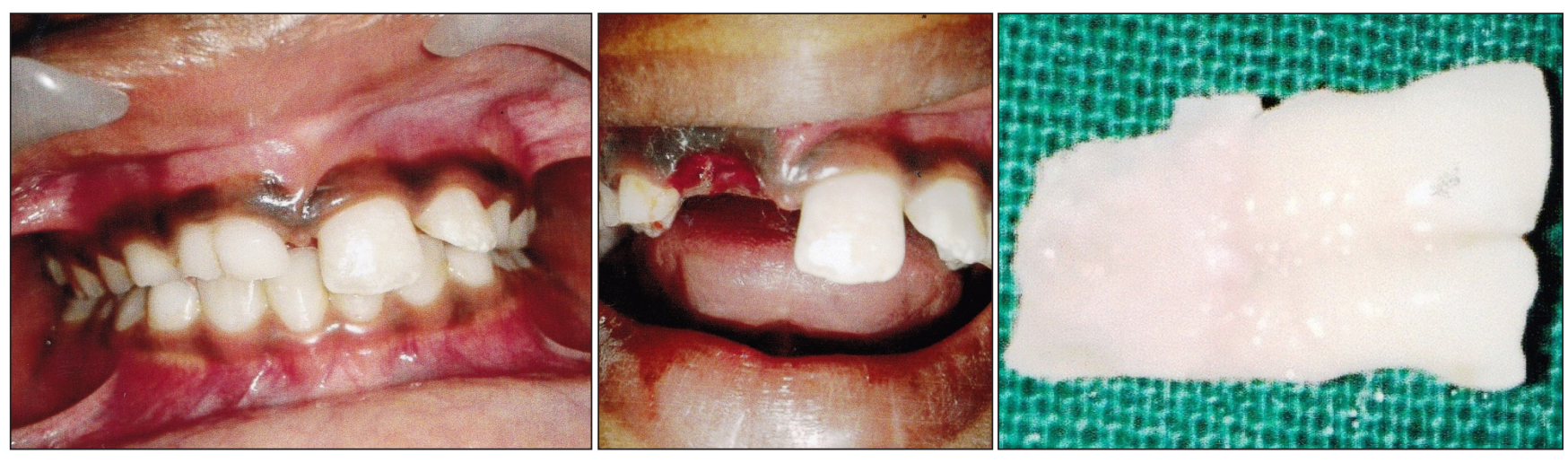

gemination. The phenomenon of gemination occurs when two teeth devel op from one single bud leading to a larger tooth. ${ }^{1,6}$ Gemination is observed in the deciduous as well as in the permanent Dentition., ${ }^{4,7}$. In the anterior region, this anomaly can cause unpleasant esthetic appearance due to irregular morphology. ${ }^{8}$

Fusion may occur between two normal teeth or between a normal tooth and a supernumerary tooth. Clinically fused anterior teeth frequently have a groove or notch on the incisal edge that goes in buccolingual direction and radiographically, the dentin of fused teeth al ways appears to be joined in some region with separate pulp chambers and canals. ${ }^{3,9}$

The etiology of fusion is still an enigma and many different views have been put forward. Shafer et al speculated that pressure produced by some physical force prol ongs the contact of the developing teeth causing fusion. ${ }^{5}$ Lowell and Soloman believe that fused teeth result from some physical action that causes the young tooth germs to come into contact, thus producing necrosis of the intervening tissue, thus allowing the enamel organ and Dental papilla to fuse together. ${ }^{6}$ Many authors have also suggested hereditary involvement as an autosomal dominant trait with reduced penetrance, trauma and environmental factors such as thalidomide, embryopathy, fetal alcohol exposure or hypervitaminosis. ${ }^{7,8}$ Others believed that fusion results from embryological persistence of the interdental lamina between 2 germs. Double teeth may also be part of syndromes such as achondrodysplasia and chondroectodermal dysplasia. ${ }^{10-13}$

Some Dental and non-Dental abnormalities have been associated with double defects. These include: supernumerary teeth, hypodontia, pegshaped permanent maxillary lateral incisors, dens in dente, nail disorders, syndactyly, successional conical, macrodontia and double permanent teeth. ${ }^{12}$, 14

Fusion may be unilateral or bilateral and most commonly occurs in primary teeth with more predilections for anterior teeth. ${ }^{8}$ The bilateral type of fusion in the primary Dentition occurs less frequently than unilateral type and is about $0.02 \% .{ }^{4}$, ${ }^{5}$ Data available for the primary dentition combined the prevalence of fused and germinated teeth, the conditions being relatively frequent, ranging from 0.5 percent to 2.5 percent according to the population surveyed. ${ }^{9-11} A$ survey of the literature has revealed prevalence estimates for bilateral double teeth ranging from 0.01 to $0.04 \%$ in the primary, and $0.05 \%$ in the permanent dentition. ${ }^{7-14}$

Gemination is more prevalent in the anterior maxillary region; whereas fusion is more commonly found in the anterior mandibular Dentition. They can also be seen unilaterally or bilaterally in either the maxillary or mandibular Dentition. ${ }^{5}$ The prevalence of tooth fusion is estimated to be $0.5 \%$ to $2.5 \%$ in the primary Dentition, whereas prevalence in the permanent Dentition seems to be lower $(0.1 \%$ to $1 \%) .{ }^{9}$ There is an overall lower incidence of double teeth in Caucasian than in Asian populations, and no differences in incidence between genders. ${ }^{11}$

Treatment objectives should aim at preserving pulpal vitality, meeting esthetic and occlusal requirements, establishing caries prevention. When fusion occurs, the clinician must be aware of following major dental concerns. ${ }^{10} \mathrm{First}$, since fused teeth are clearly wider than the surrounding teeth, esthetics may be a concern. In the present case, the 
esthetic view was a problem in primary dentition and the family was uninformed on the fused teeth until the patient visited the Dental clinic. Second, when normal teeth fuse, excess Dental space can result. This occurs because two fused teeth require less space in the Dental arch than two normal teeth. Third concern relates to both esthetics and occlusion.

The treatment usually involves Periodontics, Endodontics, selective grinding, surgical separation or Extraction followed by Prosthesis and/or Orthodontics to achieve favorable results. ${ }^{1,4,5}$ O'Reilly stated that the hemisection of two fused teeth is contraindicated because there would be exposure of the vascular canals, favoring contamination of the pulp organ and encouraging tissue necrosis. ${ }^{4}$

Generally, double teeth do not present a major clinical problem. Asymptomatic anterior double teeth should bel eft al one unless problems arise with esthetics, spacing, and caries. Simple composite restorations can be used to camouflage the double tooth and prevent caries developing in the fissures. ${ }^{3}$ Several clinical problems in the permanent Dentition follow fused primary teeth, such as physiological root resorption of fused deciduous teeth being retarded, leading to delayed or ectopic eruption of the permanent successors. ${ }^{12}$ Orthodontic management should be considered to ensure functional occlusion and advance esthetics. ${ }^{1-4}$ The best way to manage these difficult cases depends on a number of factors including the knowledge and technical skills of the practitioner.

\section{Conclusion:}

Dental anomalies are the commonest type of devel opment anomalies. When present they hamper the aesthetic and functional value. Since exfoliation times are usually different for each tooth involved in the gemination, consideration should be given to the variations in root resorption. Preservation of teeth during primary dentition period, even with uncertain prognosis, appears crucial for maintenance of the Dental arch.

Management of such condition requires a multidisciplinary approach involving two or more Dental faculties. Efforts must be directed to understand this anomaly and manage it successfully to avoid treatment complications. In present cases report, the fused primary incisors lead to delayed eruption of its successor.

They represent a challenge for clinicians because they require, in most cases, a multidisciplinary approach to achieve success and patient satisfaction. Clinicians must work together to choose the best possible treatment for the patient.

\section{References:}

1. Aguilo L, Gandia J L, Cibrian R, Catala M. Primary double teeth. A retrospective clinical study of their morphological characteristics and associated anomalies. Int J Paediatr Dent.1999; 9:175-183.

2. Carroll MK. Fusion and gemination in alternate dentitions. Oral Surg. 1990; 69: 655.

3. Maibaum WW. Fusion of confusion? Oral Surg Oral Med Oral Patho. 1990; 69:656-657.

4. O Reilly PMR. Structural and radiographic evaluation of four cases of tooth fusion. AustDent J . 1990; 35:226-229.

5. Neves AA, Neves MLA, Farinhas J A. Bilateral connation of permanent mandibular incisors: a case report. Int J Paediatr Dent. 2002; 12:61-65.

6. Nunes E, De Moraes IG, De Novaes PMO, DeSousa SMG. Bilateral fusion of mandibular second molars with supernumerary teeth: Case Report. Braz Dent J . 2002; 13:137-141.

7. Velasco LF, DeAraujoFB, Ferreira ES, Velasco LE. Esthetic and functional treatment of a fused permanent tooth: a case report. Quitessence Int. 1997; 13:677-680.

8. Milano M, Seybold SV, McCandless G,Cammarata R. Bilateral fusion of the mandibular primary incisors: report of case. ASDC J Dent Child. 1999; 66:280-282.

9. Peretz B, Brezniak N. Fusion of Primary mandibular teeth. Report of Case. ASDC J DentChild. 1992; 59:366-368.

10. Nirmala SVSG, Dedeepya P, Sivakumar Nuvvula. Supernumerary Tooth Fused with Primary Central Incisor - A Case Report. Archives of Dental Sciences 2011; 2(3):3739.

11. Hattab FN, Yassin OM and Rawashdeh MA. Supernumerary teeth: Report of three cases and review of the literature. ASDC J Dent Child 1994; 61:382-393.

12. Chunawalla $Y$, Zingade $S$, Ahmed Bijle. Pulp therapy in a maxillary fused primary central incisor and lateral incisor report of a case. IJ CD 2011; 2(2):21-24.

13. Brunet-Llobet Lluis etal. A Fused Maxillary Central Incisor and Its Multidisciplinary Treatment: An 18-Year Follow-Up. Case Reports in Dentistry, Hindawi Publishing Corporation 2014:1-6.

14. Ozant O, U mit C, Fatih A. Comprehensivetherapy of a fusion between a mandibular lateral incisor and supernumerary tooth: A case report. I Dent J 2005; 55:213-216. 